\title{
TRATAMENTO DO ABORTAMENTO INCOMPLETO POR ASPIRAÇÃO MANUAL OU CURETAGEM
}

Pedro Paulo Pereira*, André luiz Malavasi longo de Oliveira, fábio Roberto Cabar, Adriano R. Armelin, Carlos Alberto Maganha, Marcelo Zugaib Trabalho realizado na Clínica Obstétrica do Hospital das Clínicas da Faculdade de Medicina da USP

*Correspondência:

Clínica Obstétrica do Hospital das Clínicas da Faculdade de Medicina da Universidade de São Paulo

Av. Dr. Enéas de Carvalho

Aguiar, 255

$\mathrm{ICHC}, 10^{\circ}$ andar

Cerqueira César, São Paulo, SP

\begin{abstract}
RESUMO
OBjetivos. Comparar aspiração manual intra-uterina (AMIU) com curetagem uterina (D\&C) em abortamentos no primeiro trimestre no que se refere a eficiência para eliminar restos ovulares do método de aspiração manual intra-uterina com a dilatação e curetagem, ocorrência de complicações (perfuração uterina, laceração cervical, hemorragia pós-tratamento), tempo duração dos procedimentos e tempo de internaçãa das pacientes.

Métodos. Cinqüenta pacientes no grupo AMIU e 50 pacientes no grupo D\&C foram incluídas prospectivamente de maneira aleatórí. Critérios de inclusão: abortamento espontâneo, idade gestacional de até 13 semanas, colo pérvio, espessura endometrial maior que $15 \mathrm{~mm}$, estado afebril, hemoglobina superior a $10 \mathrm{~g} / \mathrm{d}$. Amostras sangüineas foram colhidas antes $\mathrm{e}$ após os procedimentos cirúrgicos para controle dos níveis de hemoglobina; anestesia foi realizada em todos os casos. 0 tempo para realização de cada procedimento cirúrgico foi cronometrado.

Resultados. Os grupos eram semelhantes quanto à idade gestacional $(9,93 \pm 2,40 ; 9,73 \pm 2,58, p 0,71)$, espessura endometrial antes da cirurgia $(22,14 \pm 4,80 ; 22,68 \pm 5,68, p$ p 0,65). Não foram observadas complicações cirúrgicas ou anestésicas em nenhum grupo. Os tempos de realização do procedimento e internação foram significativamente menores nas pacientes do grupo AMIU $(3,71 ; 10,18 \mathrm{~min}, \mathrm{p}<0,001)(14,18 ; 23,06 \mathrm{~h}, \mathrm{p} 0,03)$. 0 decréscimo nos níveis de hemoglobina após o procedimento cirúrgico foi maior no grupo $D \& C(p=0,02)$.

Conclusão. A AMIU possibilita menor perda sangüínea, requer menor tempo de realização do procedimento e menor tempo de internação hospitalar. Entretanto, ambos os procedimentos cirúrgicos mostraram-se eficientes para o tratamento de abortamentos incompletos no primeiro trimestre da gestação, não havendo complicações após a realização dos tratamentos.
\end{abstract}

UNITERMOS: Abortamento. Curetagem uterina. Aspiração manual intra-uterina. Primeiro trimestre da gestação.

\section{INTRODUÇÃO}

O abortamento é uma das intercorrências mais freqüentes da gestação e, por isso, é considerado importante problema de saúde pública, com conseqüências políticas, econômicas e sociais ${ }^{1,2,3}$. Estimase que ocorram mais de 20 milhões de abortamentos ilegais todos os anos, o que colabora sobremaneira para a morbi-mortalidade materna, principalmente em países em desenvolvimento ${ }^{2-7}$. Nesse sentido, no Brasil, onde o abortamento legal apresenta indicações muito restritas, acredita-se que o abortamento ilegal é subnotificado e, mesmo assim, alcança números de até 1,5 milhão desses procedimentos por ano ${ }^{3}$. Isso repercute sobre o ônus gerado ao Estado, porque o tratamento do abortamento incompleto, provocado ou espontâneo, acaba sendo responsável pela utilização de parte substancial de recursos do sistema público de saúde em países da América Latina4.

Esses fatos são demonstrados pelas estatísticas de hospitais da rede pública, as quais apontam que as complicações por abortamento incompleto representam uma das quatro principais causas de internação hospitalar no Brasil|2,8.

A aspiração manual intra-uterina (AMIU) tem sido usada, no mundo todo, como alternativa segura e eficaz à curetagem uterina (D\&C); esse procedimento permite a utilização instrumental de fácil manuseio e sua técnica é de simples execuçãa ${ }^{1,2,3,9,10,11}$. Além desses detalhes, outras vantagens do método são: a aceitabilidade por parte dos médicos, a satisfação das pacientes ${ }^{12,13,14}$, o uso de anestésicos locais em substituição à anestesia geral, a diminuição da permanência hospitalar e conseqüente redução de morbidade materna e dos custos hospitalares $1,2,3,15$. Lukman e Pogharian, em 1996, analisaram 1896 pacientes com indicação de esvaziamento uterino que foram submetidas a D\&C ou AMIU e concluíram que os métodos foram igualmente seguros, eficazes, simples e rápidos!.

Em 1997, Lara Ricalde et al. publicaram estudo comparando dois grupos de pacientes submetidas a esvaziamento uterino; os autores referem que a AMIU foi tão eficaz e segura quanto à D\&C, sendo, porém, mais facilmente executável e não associada a complicações graves ${ }^{22}$.

Fonseca et al., ainda em 1997, avaliaram 30 pacientes com diagnóstico de abortamento incompleto; elas foram divididas em dois grupos e cada um foi submetido a uma modalidade de tratamento. Eles concluíram que pacientes tratadas com AMIU necessitaram 77\% de tempo de internação hospitalar a menos e consumiram $41 \%$ a menos de recursos hospitalares que pacientes tratadas com $D \& C^{21}$. 
A literatura nacional é escassa em estudos que comparam a AMIU com a D\&C no tratamento do abortamento. A literatura sobre o tema é escassa em países subdesenvolvidos e existem poucos dados acerca da utilização desse método no Brasil. O objetivo desse estudo foi comparar a D\&C com a AMIU em casos de abortamento incompleto no primeiro trimestre no que se refere a eficiência para eliminar os restos ovulares do método de aspiração manual intra-uterina com a dilatação e curetagem, a ocorrência de complicações (perfuração uterina, laceração cervical, hemorragia pós-tratamento), o tempo de duração dos procedimentos e o tempo de internação das pacientes.

\section{Métodos}

O presente estudo foi realizado de modo prospectivo, no período de $I^{\circ}$ de janeiro de 2003 a 17 de março de 2004, na Clínica Obstétrica do Hospital das Clínicas da Faculdade de Medicina da Universidade de São Paulo, tendo sido aprovado pela Comissão de Ética para Análise de Projetos de Pesquisa dessa instituição.

Foram selecionadas 100 pacientes com diagnóstico de abortamento incompleto, divididas de maneira aleatória em dois grupos. Cinqüenta pacientes (grupo A) foram submetidas à D \& C e em 50 pacientes (grupo B) foi realizada a AMIU.

Foram considerados critérios de inclusão para o presente estudo: diagnóstico de abortamento espontâneo com idade gestacional menor que 13 semanas completas a partir da data da última menstruação, presença de permeabilidade do colo uterino, espessamento endometrial maior de $15 \mathrm{~mm}$ diagnosticado pela ultra-sonografia transvaginal, ausência de quadro clínico compatível com abortamento infectado (temperatura axilar superior a $37,7^{\circ} \mathrm{C}$, secreção vaginal purulenta) e concordância com assinatura do termo de consentimento pós-informado.

As pacientes selecionadas que apresentassem suspeita clínica de moléstia trofoblástica gestacional ou concentrações séricas de hemoglobina menores que $10 \mathrm{~g} / \mathrm{dL}$ foram excluídas deste estudo.

Antes do início da AMIU, as pacientes eram monitorizadas por meio de cardioscópio. Amostras de sangue eram obtidas para a monitorização de dados hematimétricos $(\mathrm{Hb} / \mathrm{Ht})$ pré e pós-procedimento.

Os tempos dispendidos para a realização dos procedimentos, assim como os tempos pré-procedimento (da admissão ao início do procedimento) e pós-procedimento (do final do procedimento à alta hospitalar) foram cronometrados.

Em ambos os procedimentos as pacientes foram acomodadas em posição ginecológica, realizada anti-sepsia da genitália com solução iodada aquosa, introdução de espéculo vaginal para visualização da cérvix, apreensão da mesma com pinça de Pozzi e, com tração delicada, retificava-se o canal cervical.

Os materiais para a aspiração manual intra-uterina consistiram de uma fonte de vácuo, tipo seringa de $60 \mathrm{ml}$ de válvula dupla, e cânulas estéreis de plástico flexível nos tamanhos (diâmetro externo): 4, 5, 6, $7,8,9,10,11$ e $12 \mathrm{~mm}$.

A AMIU era realizada pelos autores do presente trabalho da seguinte forma: após administração de analgésicos por via endovenosa (2 a $5 \mathrm{ml}$ de solução decimal contendo $100 \mathrm{mg}$ de meperidina) e confirmação do tamanho e posição do útero mediante toque combinado (essas informações eram previamente obtidas por meio de ultra-sonografia); a cânula era inserida delicadamente através da cérvix para o interior da cavidade uterina até atingir o fundo do útero. A seguir, a válvula de ajuste da seringa era aberta para transferir o vácuo, através da cânula, para o útero e, ao mesmo tempo, para aspirar o seu conteúdo por meio de movimentos de "vai-e-vem" firmes e cuidadosos da cânula.

A curetagem uterina ocorria sob anestesia geral; era realizada histerometria indireta inicial com pinça de Winter e procedia-se a retirada de restos ovulares, primeiramente com pinça de Winter e depois por meio de raspagem da cavidade endometrial com cureta fenestrada.

Foram considerados como sinais de término dos procedimentos a presença de sangue vermelho-vivo espumante e a sensação de aspereza da cavidade uterina vazia, que na AMIU também determinava limitação dos movimentos da cânula, devido a sua apreensão pelas paredes uterinas.

Após o término dos procedimentos, as pacientes eram foi mantidas em observação clínica e o material obtido (restos ovulares) era enviado para exame anatomopatológico.

Todos os dados foram anotados em um instrumento de coleta de dados que contemplava espaço para se anotar, também, as possíveis complicações observadas, como perfuração uterina e hemorragia genital.

No momento da alta hospitalar, era registrado o tempo total de permanência no hospital e recomendado retorno ambulatorial para seguimento com a mesma equipe entre 10 e 14 dias ou antes se houvesse febre, sangramento genital ou dores abdominais intensos.

Para análise dos resultados, quando havia distribuição normal das variáveis quantitativas, estas foram comparadas por meio de teste $t$ de Student. Quando essa hipótese foi rejeitada, usou-se o teste não paramétrico de Mann-Whitney, adotando-se 5\% como nível de significância. Por meio de análise de variância com medidas repetidas, os grupos foram comparados em relação ao comportamento pré e pós-operatório dos níveis de hemoglobina.

Este estudo foi realizado com a aprovação da Comissão de Ética para Análise de Projetos de Pesquisa do Hospital das Clínicas da Faculdade de Medicina da USP (CAPPesq) sob o protocolo número 250/02.

\section{Resultados}

Cinqüenta pacientes foram selecionadas aleatoriamente para cada grupo; os grupos eram semelhantes quanto à idade, tempo de amenorréia e espessura endometrial antes da realização do procedimento (Tabela I).

Ambos os grupos mostraram igual eficiência no esvaziamento uterino, não havendo necessidade de novo procedimento cirúrgico. Não foram observadas complicações em ambos os grupos (perfuração uterina, laceração cervical, complicação anestésica ou hemorragia pós-procedimento). No acompanhamento pós-operatório (até 14 dias), não houve registro de febre, dor abdominal ou hemorragia genital. 


\begin{tabular}{|c|c|c|c|}
\hline \multicolumn{4}{|c|}{$\begin{array}{l}\text { Tabela I - Caracterização dos grupos com } \\
\text { abortamento incompleto de primeiro trimestre }\end{array}$} \\
\hline & Grupo D\&C & Grupo AMIU & $p$ \\
\hline & Média $\pm d p$ & Média $\pm d p$ & \\
\hline Idade (anos) & $26,65 \pm 6,8$ & $27,50 \pm 6.9$ & 0,58 \\
\hline Atraso menstrual(semanas) & $9,73 \pm 2,6$ & $9,93 \pm 2,4$ & 0,71 \\
\hline EspessuraEco endometrial (cm) & $22,68 \pm 5,7$ & $22,14 \pm 4,8$ & 0,64 \\
\hline
\end{tabular}

$\mathrm{D \& C}=$ dilatação e curetagem

AMIU = aspiração manual intrauterina

\section{Figura I - Concentrações de hemoglobina $(\mathrm{g} / \mathrm{d})$ pré e pós-procedimento cirúrgico}

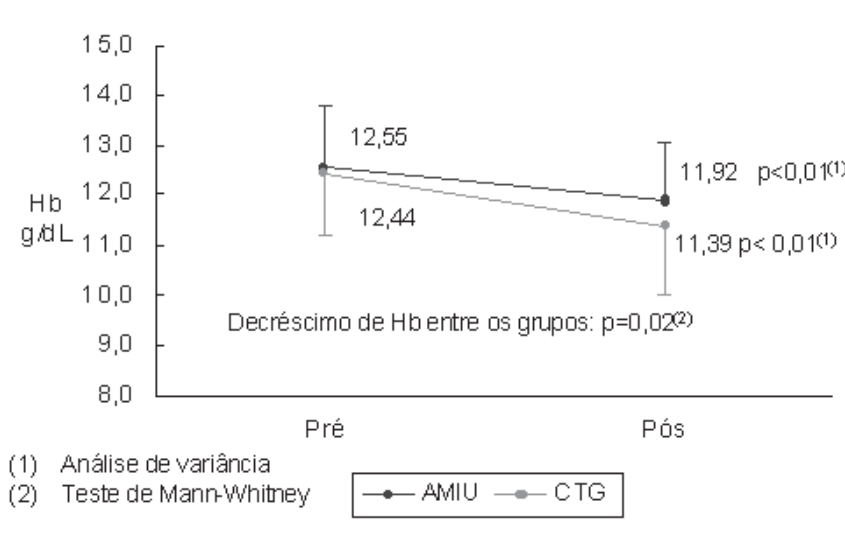

Houve diferença na duração da execução dos procedimentos entre os dois grupos. O tempo médio para o esvaziamento uterino por meio de curetagem foi de $10,18 \mathrm{~min}( \pm 6)$, enquanto que a AMIU levou, em média, 3,7I min $( \pm 1,03)(p<0,001)$.

Da mesma forma, o tempo de permanência da paciente no hospital, desde o momento de sua admissão até a alta hospitalar, foi significativamente diferente entre os dois grupos. As pacientes submetidas à curetagem uterina ficaram, em média, 23,06 horas ( $\pm 11,6)$, e as pacientes que realizaram aspiração a vácuo permaneceram internadas por 14,18 horas $( \pm 10,5)(p=0,03)$.

A diminuição das taxas de hemoglobina foi maior no grupo de pacientes submetidas à curetagem uterina, diferença que foi estatisticamente significativa $(p=0,02)$ (Figura I).

\section{Discussão}

No Hospital das Clínicas da Faculdade de Medicina da Universidade de São Paulo, como na maioria dos hospitais públicos, o método mais usado para tratamento do abortamento incompleto do primeiro trimestre (gestação de até 13 semanas) é o esvaziamento uterino por meio de curetagem, realizada sob narcose com ou sem a dilatação da cérvix (D\&C). Os riscos inerentes ao procedimento cirúrgico e anestésico, bem como a exposição das pacientes à infecção pela permanência nos hospitais, podem contribuir para o aumento da morbi-mortalidade materna e dos custos hospitalares.

O esvaziamento uterino realizado por meio de AMIU é apresentado por muitos autores como uma alternativa terapêutica, com a vantagem da substituição da anestesia geral por analgésicos ou, ainda, por bloqueio paracervical, além do encurtamento da permanência hospitalar pela maior agilidade no atendimento e precocidade da alta, o que contribuiria para a redução dos custos hospitalares ${ }^{1,2,3,15}$. Esse tratamento destina-se, portanto, também a serviços médicos de menor complexidade ou com menores recursos, como forma de melhorar os resultados e diminuir os riscos para as pacientes $1,2,3,15,16$.

A AMIU está associada a pequeno número de complicações (perfuração uterina, laceração do colo uterino, hemorragia genital), com resultados semelhantes à D\&C quanto à segurança e eficácial $1,11,17$. Westfall et al. realizaram AMIU para tratamento de abortamentos de primeiro trimestre da gestação e demonstraram eficácia de 99,5\% e ausência de maiores complicações quando realizada até a décima semana?

Neste estudo, ambos os grupos mostraram 100\% de eficácia no esvaziamento uterino. Também não observamos nenhuma complicação em ambos os grupos durante o procedimento cirúrgico (perfuração uterina, laceração cervical, complicação anestésica ou hemorragia pós-procedimento que necessitasse de tranfusão sangüínea), nem no acompanhamento pós-operatório (até I4 dias): febre, dor abdominal ou hemorragia genital abundante. Foi observado decréscimo nas taxas de hemoglobina após a realização de ambos os procedimentos, sendo maior no grupo de pacientes submetidas à curetagem uterina.

O tempo necessário para o esvaziamento uterino tem sido apontado como significativamente menor na AMIU quando comparado à $D \& C^{17}$; estudo envolvendo médicos experientes e residentes mostra que o tempo de realização do procedimento e a sensação dolorosa relatada pelas pacientes são inversamente proporcionais à experiência do cirurgião, sendo o tempo médio de 6,9 minutos ${ }^{18}$.

No presente estudo, o tempo médio para o esvaziamento uterino por meio de curetagem foi superior ao tempo do grupo tratado com AMIU (3,I vezes maior), resultado, portanto, concordante com os outros estudos.

Alguns autores sugerem que a realização da AMIU também permite menor tempo de internação hospitalar por estar relacionada a menor tempo de espera antes do procedimento, menor duração do procedimento e maior precocidade da alta hospitalar ${ }^{15}$. Fonseca et al. admitem que a realização de AMIU permite reduzir a permanência hospitalar em $77 \%$ e que esse fato representa economia de $41 \%$ nos gastos no tratamento de abortamento de primeiro trimestre ${ }^{19}$.

Também, no presente estudo, pudemos observar que o tempo de permanência hospitalar foi diferente entre os dois grupos; as pacientes submetidas à AMIU ficaram internadas, em média, 9 horas a menos que as pacientes que realizaram D\&C.

\section{ConClusão}

Os resultados apresentados neste estudo, corroborando com a literatura mundial, sinalizam como vantajosa a realização da AMIU em 
hospitais que tratam pacientes com abortamento incompleto de primeiro trimestre, inclusive naqueles de menor complexidade, representando, inequivocamente, melhoria na qualidade da assistência médica.

\section{Conflito de interesse: não há}

\section{SUMMARY}

COMPARATIVE STUDY OF MANUAL VACUUM ASPIRATION AND UTERINE CURETTAGE FOR TREATMENT OF ABORTION

OBJECTIVES. To compare manual vacuum aspiration(MVA) and uterine curettage (D\&C) for first trimester abortions, in terms of the efficiency of eliminating ovular remnants, frequency of complications, duration of the procedure, and duration of patients' hospitalization.

METHODS. In a prospective study, 50 patients in the MVA group and 50 in the D\&C group were randomly included. Inclusion criteria were: spontaneous abortion, gestational age less than 13 weeks, patent cervix, endometrialthickness $>15 \mathrm{~mm}$, afebrile state, and hemoglobin $>10 \mathrm{~g} / \mathrm{dl}$. Blood samples were collected before and after surgical procedures for control of hemoglobin levels. Anesthesia was performed in all cases. The time required for each surgical procedure was recorded.

RESULTS. Groups were similar regarding gestational age ( $9.93 \pm 2.40$ vs $9.73 \pm 2.58$ weeks; $p=0.71$ ) and endometrial thickness before surgery (22. $14 \pm 4.80$ vs $22.68 \pm 5.68 \mathrm{~mm} ; p=0.65)$. There were no surgical or anesthetic complications in either group. Durations of the procedure and of hospitalization were significantly shorter in the MVA group (3.7I vs 10.18 min, $p<0.001$, and 14.18 vs $23.06 \mathrm{~h}, p=0.03$, respectively). Decrease of hemoglobin levels was greater after the surgical procedure in the $D \& C$ group $(p=0.02)$.

CONCLUSION. MVA caused less blood loss, wasless time consuming, and resulted in shorter hospitalization. However, both surgical procedures were found to be efficientfor treatment of incomplete abortions during the first trimester of pregnancy, with no complications after both treatments. [Rev Assoc Med Bras 2006; 52(5): 304-7]

KEY WORDS: Abortion. Uterine curettage. Manual vacuum aspiration. First trimester of pregnancy.

\section{REFERÊNCIAS}

I. Lukman HY, Pogharian D. Management of incomplete abortion with manual vacuum aspiration in comparison to sharp metallic curette in an Ethiopian setting. East Afr Med J 1996;73:598-603.

2. O tratamento das complicações pós-aborto: uma intervenção para salvar a vida da mulher. Popul Rep 1997;( I0): I-28.

3. Baird TL, Gringle RE, Greenslade FC. AMIU no atendimento ao abortamento incompleto: experiência clínica e programática. Carrboro: IPAS; 1996.
4. Valera Arrunategui DR. El aborto como problema de salud en Piura metropolitana. Ginecol Obstet 1997:43:231-5.

5. Velasco Murillo V, Navarrete Hernández E, Cardona Pérez JA, Madrazo Navarro M. Mortalidad materna por aborto en el Instituto Mexicano del Seguro Social (1987 a 1996). Rev Méd IMSS 1999; 37:73-9.

6. Muñoz L, Ñañez H, Becerra E, Klevens J. Mortalidad materna Instituto Materno Infantil 1976-1980. Rev Colomb Obstet Ginecol 1985;36:227-43.

7. Donoso Sina E, Poblete L A, Villarroel del Pino L. Mortalidad materna: Chile 1990- 1996. Rev Chil Obstet Ginecol 1998;63:290-7.

8. Parpinelli MA, Surita FGC, Pereira BG, Cecatti JG. Mortalidade materna na cidade de Campinas, no período de 1992 a 1994. Rev Bras Ginecol Obstet 1999;21:227-32

9. Westfall JM, Sophocles A, Burggraf H, Ellis S. Manual vacuum aspiration for first-trimester abortion. Arch Fam Med 1998;7:559-62.

10. Suarez Rincon AE, Arévalo Lagunas I, Cerpa Batres MG, Díaz Rodriguez MC. Validación diagnóstica de la aspiración manual endouterina para la detección de hiperplasia y cáncer de endométrio en pacientes con sangrado uterino anormal. Ginecol Obstet Méx 2000;68: I-6.

I I. Hemlin J, Moller B. Manual vacuum aspiration, a safe and effective alternative in a early pregnancy termination. Acta Obstet Gynecol Scand 2001;80:563-7.

12. Bird ST, Harvey SM, Nichols MD, Edelman A. Comparing the acceptability of manual vacuum aspiration and electric vacuum aspiration as methods of early abortion. J Am Med Womens Assoc 200 I;56: I24-6.

13. Leme VM, Mtimavalye LA, Thole GC, Mvula MT. The impact of the manual vacuum aspiration (MVA) technique on health care services at Queen Elisabeth Central Teaching Hospital, Blantyre, Malawi. S Afr Med J 1997;87:218-22, 224.

14. Creinin MD. Randomized comparison of efficacy, acceptability and cost of medical versus surgical abortion. Contraception 2000;62: 1 17-24.

15. Magotti RF, Munjinja PG, Lema RS, Ngwalle EK. Cost-effectiveness of managing abortions: manual vacuum aspiration (MVA) compared to evacuation by curettage in Tanzania. East Afr Med J 1995;72:248-5I.

16. Kizza AP, Rogo KO. Assessment of the manual vacuum aspiration (MVA) equipment in the management of incomplete abortion. East Afr Med J 1990;67:812-22.

17. Kullier R, Fekih A, Hofmeyer GJ, Campana A. Surgical methods for first trimester termination of pregnancy. Cochrane Database Syst Rev $2001 ;(4):$ CD002900.

18. Edelman A, Nichols MD, Jensen J. Comparison of pain and time of procedures with two first-trimester abortion techniques performed by residents and faculty. Am J Obstet Gynecol 200 I; | 84: I 564-7.

19. Fonseca W, Misago C, Fernandes L, Correia L, Silveira D. Uso da aspiração manual a vácuo na redução do custo e duração de internamentos por aborto incompleto em Fortaleza, CE, Brasil. Rev Saúde Pública 1997;31:472-8. Aceito para publicação: | 3// |/2005 